\title{
Determination of fractal dimension of physiologically characterized neurons in two and three dimensions
}

\author{
F. Caserta ${ }^{\text {a }}$, W.D. Eldred ${ }^{\text {b,* }}$, E. Fernandez ${ }^{\text {c }}$, R.E. Hausman ${ }^{\text {b }}$, L.R. Stanford ${ }^{d}$, \\ S.V. Bulderev a , S. Scilwarzer a , H.E. Stanley ${ }^{a}$ \\ a Center for Polymer Studies and Department of Physics, Boston University, Boston, MA 02215, USA \\ ${ }^{\mathrm{h}}$ Department of Biology, Boston University, Boston, MA 02215, USA \\ ${ }^{c}$ Department of Histology, Medical Faculty of the University of Alicante, 03080 Allcante, Spain \\ ${ }^{d}$ Department of Comparative Biosciences, and the Waisman Center on Mental Retardation, Unicersity of Wisconsin, Madison, Wisconsim 53706, USA
}

Received 18 July 1991; revised 1 June 1994; accepted 2 June 1994

\begin{abstract}
Although there is a growing interest in the application of fractal analysis in neurobiology, questions about the methodology have restricted its wider application. In this report we discuss some of the underlying principles for fractal analysis, we propose the cumulative-mass method as a standard method and we extend the applicability of fractal analysis to both 2 and 3 dimensions. We have examined the relationship between the method of $\log -\log$ Sholl analysis and fractal analysis and have found that they correlate well. Measurements of physiolngically characterized retinal ganglion cells indicate that different cell types can have significantly different fractal dimensions. Such differences may allow the correlation of the physiological type of a neuron with its morphological fractal dimension.
\end{abstract}

Keywords: Retinal ganglion cell; Cumulative-mass method; Physiologically characterized neuron; Fractal dimension; Sholl analysis: Dendritic branching

\section{Introduction}

A major goal in neurobiology has been the meaningful morphological analysis of neuronal arborization patterns, but only a few methods such as Sholl analysis (Sholl, 1953) have achieved wide application. Recently, in addition to Sholl analysis, researchers have used fractal analysis to quantify neuronal dendritic arborizations. Fractal analysis has also been used to describe how completely a neuron fills its dendritic field or the straightness of individual dendrites (Montague and Friedlander, 1991). However, previous studies have used the following different methods for calculating the fractal dimension $\left(d_{\mathrm{f}}\right)$ of a neuron: cumulative-mass (Caserta et al., 1990), box counting (Morigiwa et al., 1989) and coastline measurement (Smith et al., 1989). The use of different methods of fractal analysis has

\footnotetext{
* Corresponding author. Tel.: (617) 353-2439; Fax: (617) 353-6340.
}

made comparison of results difficult because each method of determination can give slightly different results when used to analyze the same structure. Here we suggest the adoption of the cumulative-mass method which has been shown to be more accurate than box counting (Li et al., 1989; Tel et al., 1989). Furthermore, we have attempted to describe many of the underlying assumptions and limitations of these techniques when they are applied to biological preparations. In this article we try to improve on existing methods of morphological analysis in four different ways. First, we review the method of Sholl analysis which has long been used for quantitative morphological studies of the dendritic arborizations of neurons (Sholl, 1953). Second, we compare Sholl analysis with fractal analysis (Caserta et al., 1990) for the characterization of neuronal arborizations. To do this, we compare the fractal dimension obtained using the cumulative-mass method, with the results of both $\log -\log$ and semi-log Sholl analysis (Sholl, 1953) for the same neurons. We ask if one method is better than the other and if they are 
mathematically related. Third, we extend the method of fractal analysis, which is traditionally a 2-dimensional (2-D) method, to 3 dimensions which is more appropriate for most types of neurons. We did this by analyzing several neurons in both 2 and 3 dimensions using the cumulative-mass method, and comparing the results. Finally, previous fractal analysis studies have largely been performed on neurons whose physiological properties have not been directly assessed (Montague and Friedlander, 1989, 1991; Morigiwa et al., 1989; Smith et al., 1989; Caserta et al., 1990). This left open the question of whether there is a correlation between the fractal dimension and physiology of a given cell. To examine this possibility, we compare the fractal dimensions of 3 physiologically distinct classes of cat retinal ganglion cells.

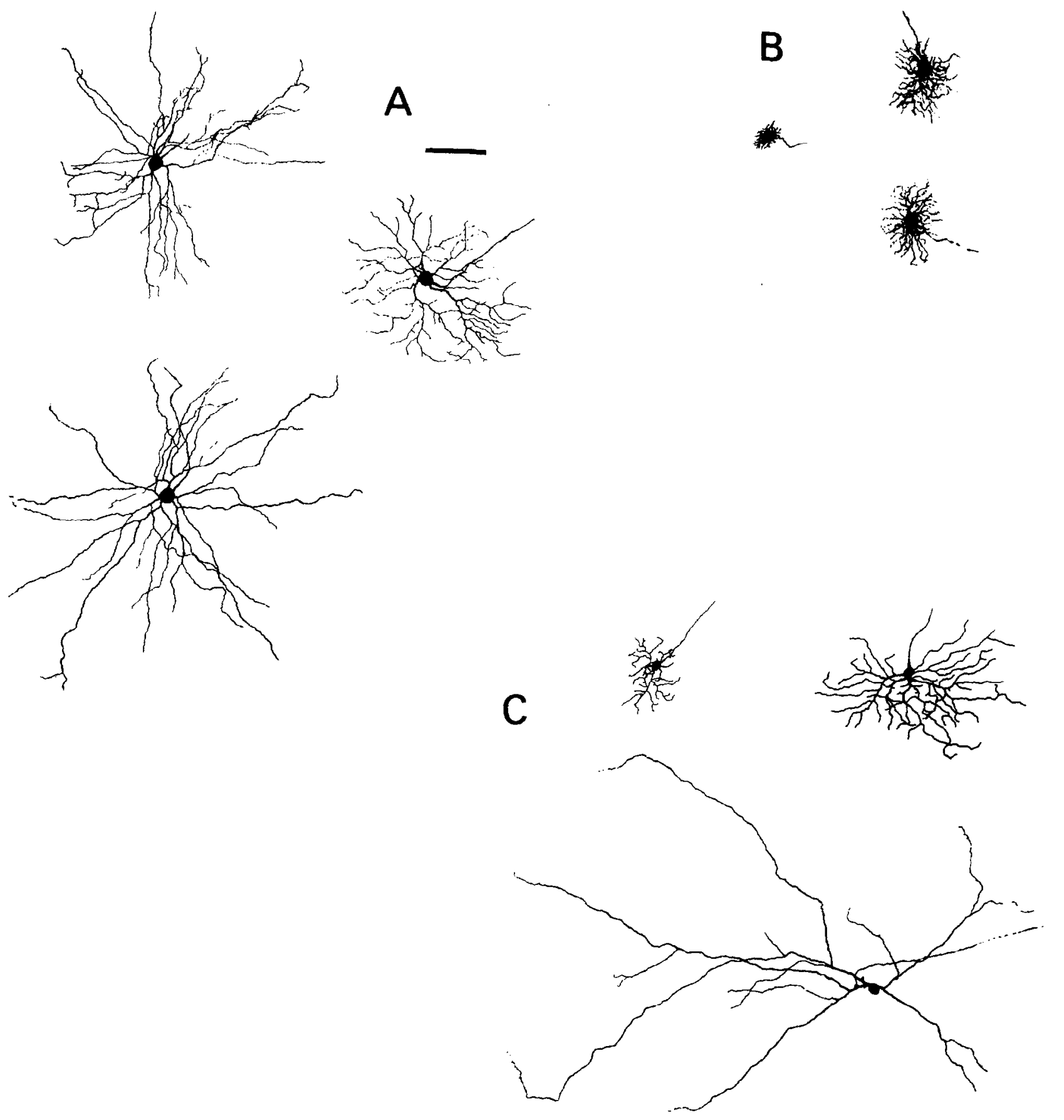

Fig. 1. Line drawings of 9 of the HRP-injected, functionally identified retinal ganglion cells that we analyzed. A: 3 tonic W cells. B: 3 X cells. C: phasic $W$ cells. Both the $X$ cells (B) and tonic $W$ cells (A), although varying in size (generally as a function of retinal location), have dendritic arbors that are relatively homogeneous. In contrast, phasic $W$ cells have very heterogeneous dendritic arbors, ranging in form from highly branched and compact to wide and sparse. Scale bar: $100 \mu \mathrm{m}$. 


\section{Materials and methods}

\subsection{Methodology for fractal analysis}

The method of fractal analysis described here can be standardized and extended to 3 dimensions. It includes image acquisition, cumulative-mass fractal dimension calculation, and curve fitting. Image acquisition was performed using an Applescanner (resolution of $637 \times 825$ pixels) to digitize camera lucida line drawings of horseradish peroxidase (HRP) injected retinal ganglion cells. This improved on our earlier work (Caserta et al., 1990) which used a video camera with a resolution of $256 \times 256$ pixels. Images of neurons (Fig. 1A,B,C) digitized by the Applescanner (Fig. 2A,D,G) were input into a program written in FORTRAN which used the cumulative-mass method to determine the fractal dimension. This program produced a plot of the natural $\log$ of the mass versus the natural $\log$ of the radius (Fig. 2B,E,H). The cumulative-mass fractal dimension $d_{\mathrm{f}}$ of the digitized patterns was determined as follows. First, we randomly chose a point belonging to

2
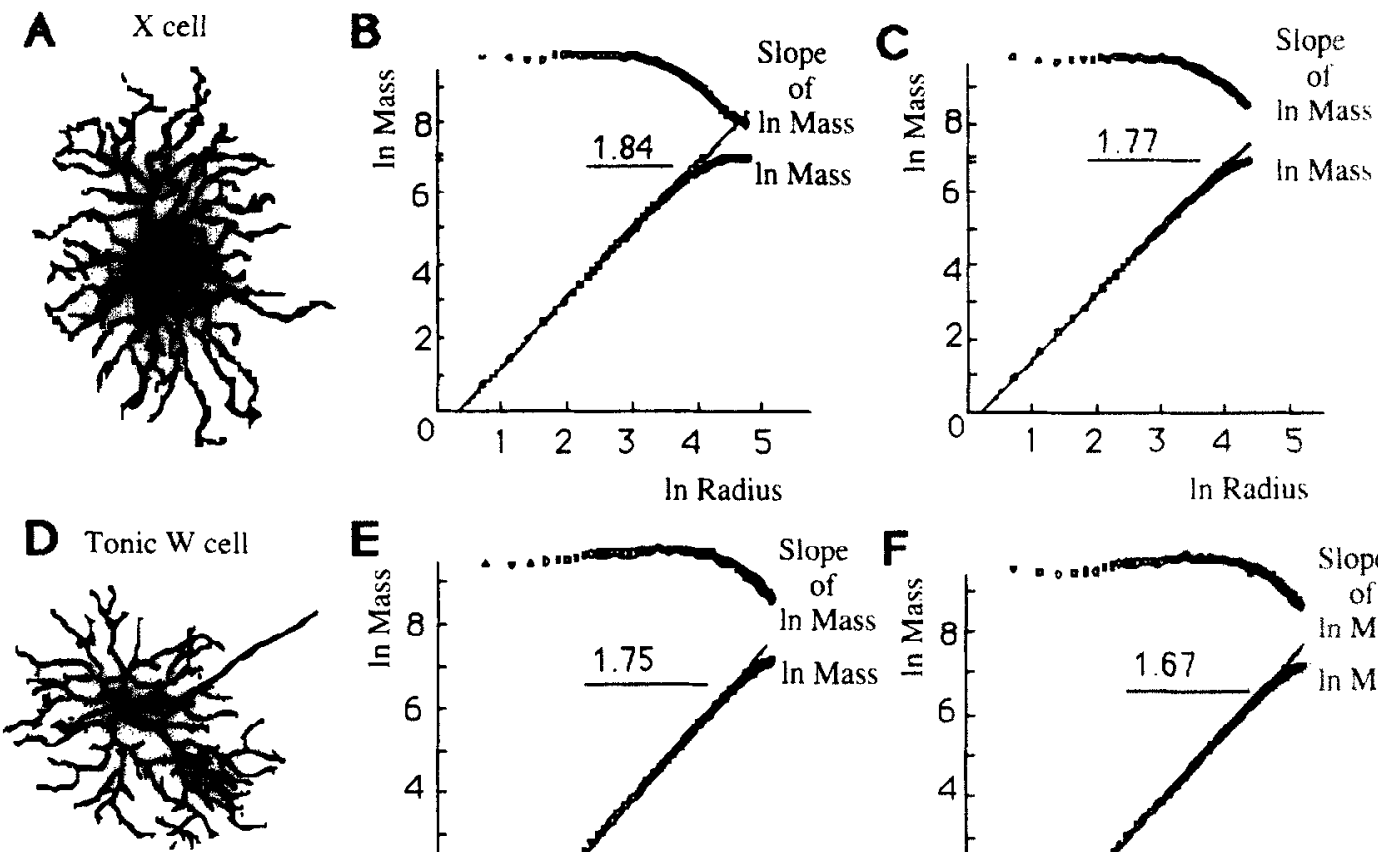

E
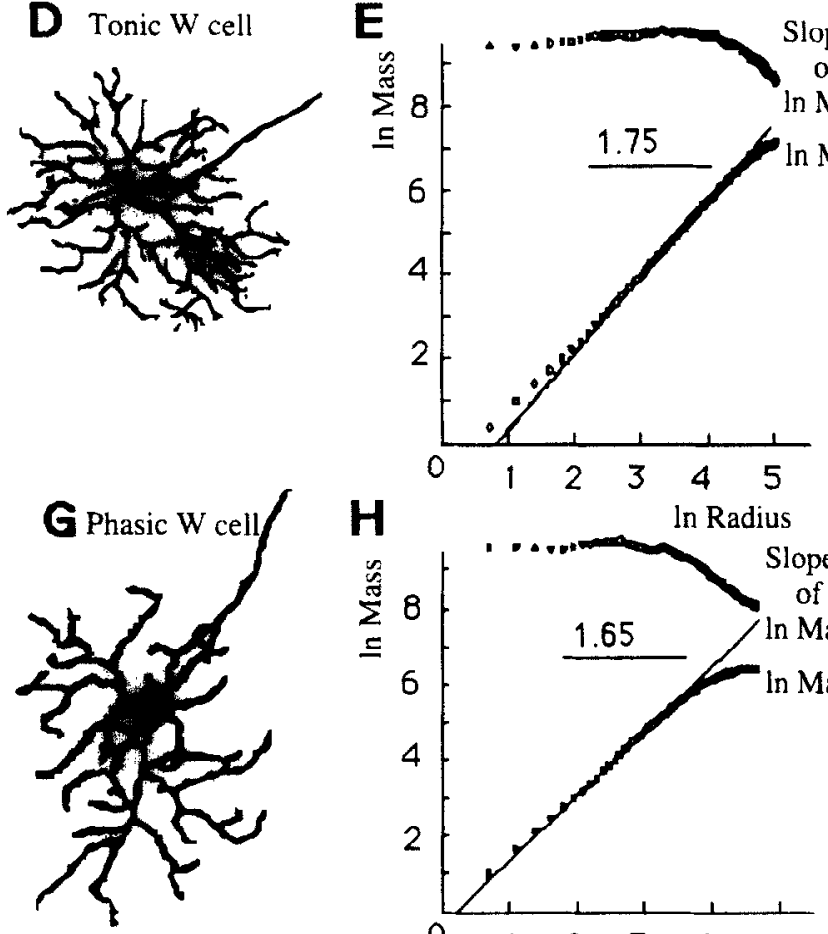

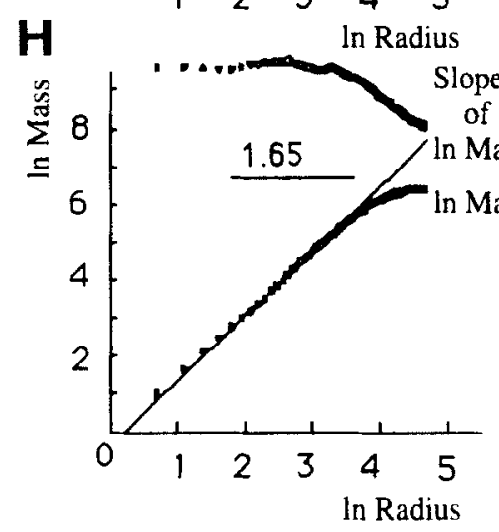

3-Dimensional $\mathrm{d}_{\mathrm{f}}$

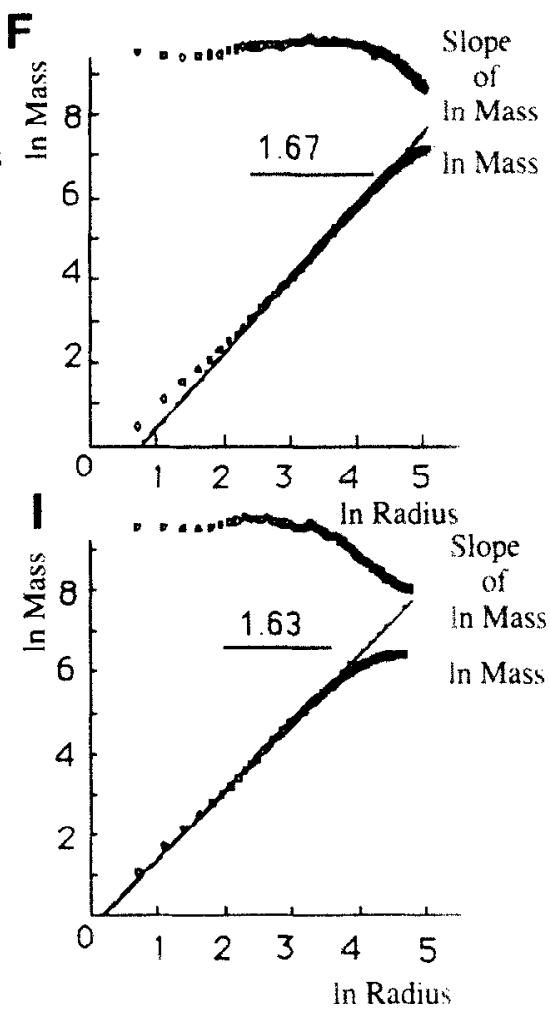

2-Dimensional $\mathrm{d}_{\mathrm{f}}$

Fig. 2. Digitized cell images are shown with their corresponding 3-D and 2-D fractal analyses. In the fractal analysis graphs, natural log of the mass is plotted against natural log of the radius in the lower plot and the slope of the lower plot is plotted in the upper plot. a: digitized image of an X cell. b: 3-D fractal analysis of Fig. 2a. c: 2-D fractal analysis of Fig. 2a. d: digitized image of a tonic W cell. e: 3-D fractal analysis of Fig. 2d. f: 2-D fractal analysis of Fig. 2d. g: digitized image of a phasic W cell. h: 3-D fractal analysis of Fig. 2g. i: 2-D fractal analysis of Fig. $2 \mathrm{~g}$. In all the fractal analyses, the horizontal bars indicate the range over which the fractal dimension, listed above it. was calculated. 
the digitized neuron as a 'local origin' (center). We constructed a sequence of concentric disks around this local origin, each with a different radius, $r$. For each disk, we counted all the pixels $N(r)$, which were given by the total number of pixels belonging to the neuronal shape in that disk. We then calculated $M(r)$ as the average of $N(r)$ over all possible local origins within the radius of gyration of the object. It is necessary to sample all local origins to eliminate potential inaccuracies which might result from some parts of the object possessing higher or lower fractal dimensions than others. The radius of gyration of an object is the square root of the sum of the squares of the radii from the center of mass to all the points on the object, divided by the square root of the number of points. For a fractal object, the mathematics predict $M(r)$ to scale as $M(r) \sim r^{d_{\mathrm{f}}}$ (Vicsek, 1989). Thus the slope of a double logarithmic plot of $M(r)$ against $r$ gives a quantitative value of $d_{\mathrm{f}}$. By definition, the linear region of this plot is the region in which the local slope is constant as a function of radius. In order to determine the location of the linear region, we calculated the local slopes of the plot. The region in which the local slopes were found to be constant was taken as the linear region. The 2-point local slopes were calculated as the difference in $\ln M$ divided by the difference in $\ln r$ for every 2 successive points. The 8-point local slopes were calculated as the slope of a least-squares line fit to every 8 successive points. This protocol has been successfully applied to a wide range of fractal objects (Nittmann et al., 1985; Daccord et al., 1986; Daccord, 1987; Daccord and Lenormand, 1987). To extend these methods to 3 dimensions, we counted all the mass (pixels) inside spheres of various radii instead of disks.

For comparison, we also analyzed neurons using the box-counting method (Morigiwa et al., 1989) which covers the image with boxes of various sizes and the coastline method. The coastline method is equivalent to using the box-counting method to analyze only the border outline or coastline of the neuron. The coastline and box-counting fractal dimensions were calculated using the IMAGE program developed at the National Institutes of Health for the Macintosh computer. This program is available by anonymous FTP from the Internet address, ZIPPY.NIMH.NIH.GOV [128.231.98.32] (enter 'anonymous' for the user name and anything you like for the password.)

When analyzing a neuron's fractal dimension using box-counting or the cumulative-mass method, one must fit a line to the graph of mass versus radius which is non-linear. Some analysis programs such as the IMAGE program we employed for box counting, choose the linear range for the user, making it impossible to estimate the effects of the non-linearity. In order to eliminate variability due to different choices of linear ranges for different cells (P. Meakin, personal commu-

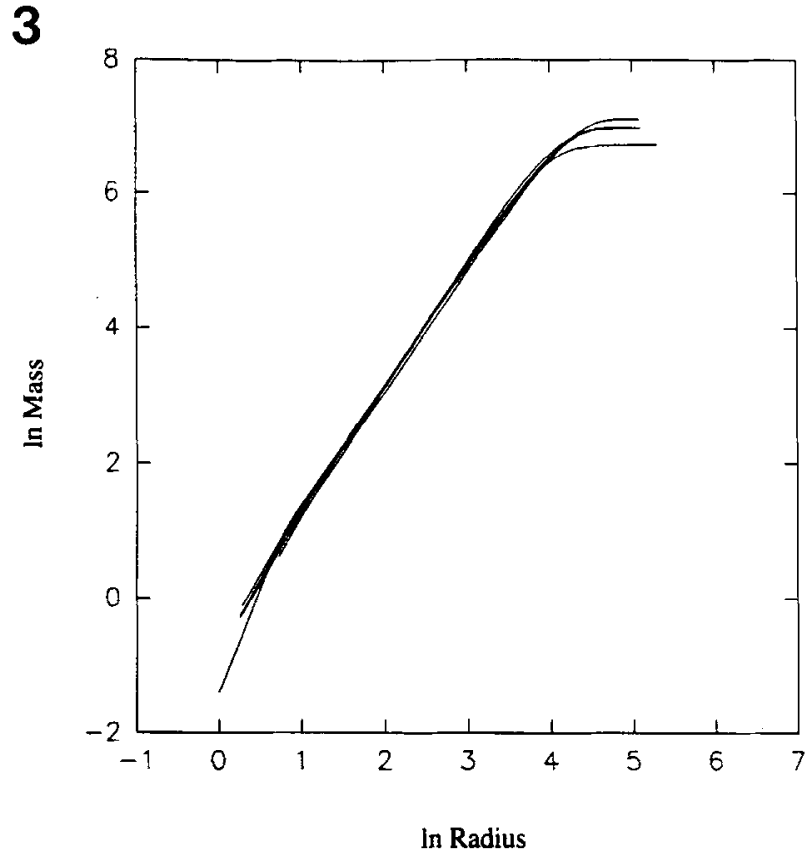

Fig. 3. Graph showing the superposition of the $\log M$ versus $\log r$ graphs for $5 \mathrm{X}$ cells. The graph demonstrates that the scaling behavior of $M$ versus $r$ is very similar for each cell.

nication) we plotted the $M(r)$ for cells of the same physiological type over one another (Fig. 3) and then chose a single linear range for all cells of a given type.

\subsection{3-Dimensional fractal analysis method}

We extended the same 2-D method detailcd above to 3 dimensions as follows. 3-D data sets of $x, y$, and $z$ coordinates were obtained using a Eutectic Electronics 3-D Reconstruction System (Eutectic Electronics, Raleigh, NC) for several of the neurons we studied. To check the validity of approximating these neurons as 2-D, we calculated the fractal dimension of each of these neurons both as a 3-D object (using $x, y$, and $z$ coordinates) and as a 2-D projection (using only $x$ and $y$ coordinates with the $z$ coordinates set uniformly to zero).

The same cumulative-mass algorithm was applied to both 2-D as well as 3-D data. The only change necessary was that for 2 dimensions the distance of a pixel from the center of a disk is the square root of $\left(x^{2}+y^{2}\right)$, but for 3 dimensions the distance of a pixel from the center of a sphere is the square root of $\left(x^{2}+y^{2}+z^{2}\right)$. Once this distance was calculated, the rest of the algorithm simply found all pixels less than a given radius from the center, averaged over all centers on the neuron and plotted mass as a function of radius.

\subsection{Comparison of fractal and Sholl analysis methods}

For Sholl analysis, images of the identified cells were obtained as described above. Our method of 
Table 1

Comparison of $d_{\mathrm{f}}$ for different physiological cell types using 2-D and 3-D fractal analysis, and Sholl analysis

\begin{tabular}{llll}
\hline & $\begin{array}{l}\text { X cells } \\
\left(\text { mean } d_{\mathrm{f}} \pm \mathrm{SD}\right)\end{array}$ & $\begin{array}{l}\text { Tonic W cells } \\
\left(\text { mean } d_{\mathrm{f}} \pm \mathrm{SD}\right)\end{array}$ & $\begin{array}{l}\text { Phasic W cells } \\
\left(\mathrm{mean} d_{\mathrm{f}} \pm \mathrm{SD}\right)\end{array}$ \\
\hline 2-D cumulative mass & $1.75 \pm 0.02$ & $1.62 \pm 0.02$ & $1.52 \pm 0.15$ \\
3-D cumulative mass & $1.80 \pm 0.04$ & 1.73 & $1.49 \pm 0.21$ \\
Sholl analysis & $1.86 \pm 0.15$ & $1.70 \pm 0.32$ & $1.62+0.20$ \\
2-D box counting (coastline) & $1.60 \pm 0.08$ & $1.55 \pm 0.07$ & $1.47 \pm 0.12$ \\
2-D box counting (no coastline) & $1.54 \pm 0.09$ & $1.47 \pm 0.06$ & $1.37 \pm 0.13$ \\
3-D box counting & $1.81 \pm 0.14$ & 1.50 & $1.52+0.12$ \\
\hline
\end{tabular}

Sholl analysis consisted of counting the intersections of dendrites with circles of various radii, which were centered on the cell body, plotting the number of intersections versus radius and fitting straight lines to these curves. We plotted the number of intersections as a function of radius including both the log of intersections/linear radius and the log of intersections/log of radius. From these plots we determined the slopes using the least-squares method.

\subsection{Physiological characterization of identified cat reti- nal ganglion cells}

We analyzed physiologically characterized cat retinal ganglion cells to determine if the fractal dimension of a cell was related to its physiology. The electrophysiological and neuroanatomical methods used for the recording and injection of the cells, and their qualitative morphological analysis have been described previously (Stanford, 1987a,b). Briefly, glass micropipettes filled with HRP were used to record from retinal ganglion cells in vivo. Based on their responses to visual stimuli and the conduction properties of their axons, the neurons were classified as $\mathrm{X}$ cells, tonic $\mathrm{W}$ cells, or phasic W cells and subsequently injected intracellularly with HRP. After the animal was killed, the retinae were dissected from the sclera and pigment epithelium, and reacted in a solution of nickel and cobalt-enhanced 3,3'-diaminobenzidine to reveal the HRP-labeled ganglion cells (Adams, 1981). Retinal whole mounts were used to make line drawings of the recovered neurons using a $\times 100$ oil objective (N.A. $=$ 1.32) and a camera lucida attachment (see Fig. 1).

For the present study, we analyzed drawings of 20 physiologically identified retinal ganglion cells $(9 \mathrm{X}$ cells, 6 tonic $\mathrm{W}$ cells, and 5 phasic $\mathrm{W}$ cells) using several methods to determine their fractal dimensions. We analyzed the significance of differences between the mean $d_{\mathrm{f}} \mathrm{s}$ for the specific cell types using Student's $t$ test, 1-way ANOVA, Fisher's PLSD and Scheffé's test (Tables 4-7).

\section{Results}

\subsection{Standardization of fractal analysis}

The results of 2-D cumulative-mass fractal dimension calculations for the 3 types of retinal ganglion cells ( $\mathrm{X}$, tonic $\mathrm{W}$, and phasic $\mathrm{W}$ ) in 2 dimensions (averaging 1.75 SD 0.02, 1.62 SD 0.02 and 1.52 SD 0.15, respectively) and 3 dimensions (averaging 1.80 SD 0.04, 1.73 and 1.49 SD 0.21 , respectively), are shown in Table 1. Typical cells are shown in Fig. 1 and fractal analysis graphs of selected neurons are shown in Fig. 2.

In a previous report (Caserta et al., 1990), our algorithm for fractal dimension calculation was called box counting. However, box counting usually refers to covering an object with a minimal number of boxes, rather than counting the number of pixels in a disk, as in our previous and present algorithm. We now apply the more descriptive term 'cumulative-mass method' because we actually counted the number of pixels needed to cover the part of the image inside a disk of a given size. The cumulative-mass method is more accu-

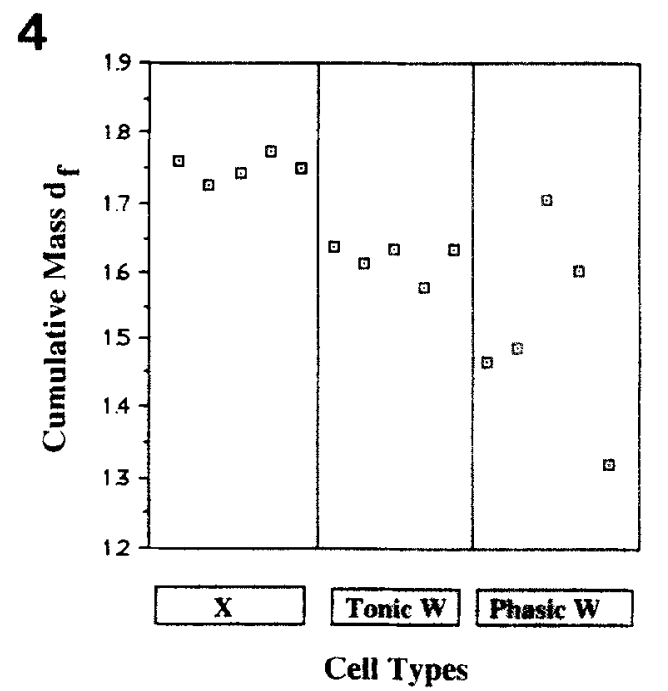

Fig. 4. $d_{i}$ for each neuron analyzed by the $\left.2-1\right)$ cumulative-mass method in this study. 
rate than true box counting (covering the image with boxes) (Tel et al., 1989; $\mathrm{Li}$ et al., 1989) because boxcounting analyzes the artifactual, low $d_{\mathrm{f}}$ 'unfinished' periphery as well as the high $d_{\mathrm{f}}$ 'finished' region, while the cumulative-mass method analyzes only the 'finished' region. In the limit where the size of the fractal object goes to infinity, the fraction of the fractal object which is 'finished' is unity and both methods converge to the same answer. Further comments on the mathematical significance of 'finished' versus 'unfinished' are included in the discussion.

\subsection{2-D versus 3-D fractal analysis}

We compared the 2-D and 3-D analyses of the same cells (see Table 1). We found $d_{\mathrm{f}} \mathrm{s}$ to be $4.9 \%$ higher, on average, for 3-D than for 2-D analysis of the same cell.

\subsection{Analysis of the fractal dimensions physiologically different cell types}

We analyzed 3 types of cat retinal ganglion cells (X, tonic $W$, and phasic $W$ ) using the 2-D cumulative-mass method (Fig. 4) and found their combined average fractal dimensions (1.63 SD 0.13 ) to be near the range of fractal dimensions produced by diffusion-limited-aggregation (DLA) in 2-dimensions (1.70 SD 0.10) (Caserta et al., 1990). Although the box-counting method gives lower fractal values than the cumulativemass method (Table 2), the relative differences between the cell types were consistent in that the $\mathrm{X}$ cells had the highest fractal values, the tonic $\mathrm{W}$ cells had intermediate values and the phasic $\mathrm{W}$ cells had the lowest average values. The phasic $\mathrm{W}$ cells also had the highest variability in fractal dimension using either the cumulative-mass or box-counting methods.

To investigate if there were significant differences between the fractal dimensions of each type of ganglion cell, we used the Student's $t$ test (Table 3) and analysis of variance (ANOVA) (Table 4). For X cells versus tonic $\mathrm{W}$ cells, only the 2-D cumulative-mass method showed a significant difference between these 2 physiological types. For $\mathrm{X}$ cells versus phasic W cells, all the fractal methods, but not Sholl analysis, showed a significant difference between the 2 physiological types. For tonic W cells versus phasic W cells, none of the methods showed a significant difference between the 2 physiological types. Also, our results indicated that $\mathrm{X}$ cells and phasic W cells had the greatest differences in fractal dimension (Table 1). The 2-D boxcounting method (no coastline) seemed to be more useful than 2-D box counting (coastline) because it showed larger significant differences between $X$ cells and phasic W cells (Table 3 ).

We used a 1-way ANOVA to investigate differences between means. The results showed that there were significant differences in the mean fractal dimension of $\mathrm{X}$ cells, tonic $\mathrm{W}$ cells, and phasic $\mathrm{W}$ cells using 2-D cumulative-mass, 3-D cumulative mass, and 2-D boxcounting methods (no coastline), as shown in Table 4. There were no differences between the electrophysiologically defined cat ganglion cells using either Sholl

Table 2

Values of $d_{\mathrm{f}} \mathrm{s}$ and Sholl parameters by physiological cell type

\begin{tabular}{lllll}
\hline & Mean $d_{\mathrm{f}}$ & $\mathrm{SD}$ & $d_{\mathrm{f}}$ Range & Sample size \\
\hline X cells & & & & 5 \\
2-D cumulative mass & 1.75 & 0.02 & $1.72-1.77$ & 5 \\
3-D cumulative mass & 1.80 & 0.04 & $1.75-1.85$ & 5 \\
Sholl analysis & 1.86 & 0.15 & $1.64-1.99$ & 9 \\
2-D box counting (coastline) & 1.60 & 0.08 & $1.44-1.70$ & 8 \\
2-D Box counting (no coastline) & 1.54 & 0.09 & $1.34-1.62$ & 5 \\
3-D box counting & 1.81 & 0.14 & $1.66-1.97$ & 5 \\
Tonic W cells & 1.62 & 0.02 & $1.58-1.64$ & 5 \\
2-D cumulative mass & 1.73 & $\mathrm{NC}$ & $1.73-1.73$ & 6 \\
3-D cumulative mass & 1.70 & 0.32 & $1.38-2.14$ & 5 \\
Sholl analysis & 1.55 & 0.07 & $1.44-1.63$ & 1 \\
2-D box counting (coastline) & 1.47 & 0.06 & $1.39-1.56$ & \\
2-D box counting (no coastline) & 1.50 & $\mathrm{NC}$ & $1.50-1.50$ & \\
3-D box counting & & & \\
Phasic W cells & 1.52 & 0.15 & $1.32-1.70$ & 5 \\
2-D cumulative mass & 1.49 & 0.21 & $1.34-1.64$ & 2 \\
3-D cumulative mass & 1.62 & 0.20 & $1.44-1.95$ & 5 \\
Sholl analysis & 1.47 & 0.12 & $1.35-1.63$ & 5 \\
2-D box counting (coastline) & 1.37 & 0.13 & $1.25-1.56$ & 5 \\
2-D box counting (no coastline) & 1.52 & 0.12 & $1.43-1.60$ & 2 \\
3-D box counting & & & \\
\hline
\end{tabular}

Mean, SD and range (minimum-maximum) are shown for each type of cat retinal ganglion cell studied. All the values are rounded up to 2 decimal digits. $\mathrm{NC}=$ the SDs were not computed because of the limited number of cells. 
Table 3

Comparisons of different physiological cell types using different fractal methods and Sholl analysis using the Student's $t$ test (2-tailed)

\begin{tabular}{llrl}
\hline Method & $t$ valuc & $d_{\mathrm{f}}$ & $P$ \\
\hline Method (X cells versus tonic W cells) & & & \\
2-D cumulative mass & 9.38 & 8 & 0.001 \\
3-D cumulative mass & $\mathrm{NC}$ & & \\
Sholl analysis & 1.00 & 8 & $\mathrm{NS}$ \\
2-D box counting (coastline) & 1.18 & 13 & $\mathrm{NS}$ \\
2-D box counting (no coastline) & 1.51 & 11 & $\mathrm{NS}$ \\
3-D box counting & $\mathrm{NC}$ & & \\
Method (X cells versus phasic W cells) & & \\
2-D cumulative mass & 3.56 & 8 & $<0.007$ \\
3-D cumulative mass & 3.68 & 5 & $<0.014$ \\
Sholl analysis & 2.08 & 8 & $\mathrm{NS}$ \\
2-D box counting (coastline) & 2.38 & 12 & $<0.035$ \\
2-D box counting (no coastline) & 2.79 & 11 & $<0.017$ \\
3-D box counting & 2.65 & 5 & $<0.046$ \\
Method (tonic W cells versus phasic W cells) & & \\
2-D cumulative mass & 1.58 & 8 & NS \\
3-D cumulative mass & $\mathrm{NC}$ & & \\
Sholl analysis & 0.45 & 8 & $\mathrm{NS}$ \\
2-D box counting (coastline) & 1.36 & 9 & $\mathrm{NS}$ \\
2-D box counting (no coastline) & 1.53 & 8 & $\mathrm{NS}$ \\
3-D box counting & $\mathrm{NC}$ & & \\
\hline
\end{tabular}

NC, not computed; $d f$, degrees of freedom; 'NS', $P>0.200$.

analysis, 2-D box counting (with coastline) or 3-D box counting. The results of comparisons using the 3-D box-counting method were limited by lack of data in that there was only 1 tonic $W$ cell and 2 phasic $W$ cells.

We also used the Fisher's PLSD test and the Scheffé test to analyze the differences in fractal dimension of the different cell types (Table 5). The 2-D cumulativemass method seems to be the most successful in distinguishing between physiologically characterized ganglion cells. To determine whether the difference between the fractal values resulting from the use of different methods was significant, we used a $t$ test for matched pairs (Table 6). We found that the 2-D and 3-D cumulative-mass methods generally had significantly different means from each other, and the means with the 2-D cumulative-mass method were different from those of both the 2-D box-counting methods. The Sholl and 3-D cumulative-mass methods both had significant differences in means from 2-D box counting

Table 4

Distinguishing different physiological cell types types using different fractal methods and Sholl analysis using 1-way ANOVA analysis

\begin{tabular}{lll}
\hline Method & $F$ ratio & $P$ \\
\hline 2-D cumulative mass & 9.29 & $<0.003$ \\
3-D cumulative mass & 6.77 & 0.030 \\
Sholl analysis & 1.30 & $\mathrm{NS}$ \\
2-D box counting (coastline) & 3.23 & $\mathrm{NS}$ \\
2-D box counting (no coastline) & 4.70 & $<0.026$ \\
3-D box counting & 4.76 & $\mathrm{NS}$ \\
\hline
\end{tabular}

NS, the method could not distinguish between different classes of cells $(P>0.20)$.
Table 5

Distinguishing different physiological cell types types using different fractal methods and Sholl analysis using the Fisher PLSD and Scheffé tests

\begin{tabular}{llll}
\hline Method & $\begin{array}{l}\text { X vs. tonic } \\
\text { W cells }\end{array}$ & $\begin{array}{l}\text { X vs. } \\
\text { phasic } \\
\text { W cells }\end{array}$ & $\begin{array}{l}\text { Tonic W vs. } \\
\text { phasic } \\
\text { W cells }\end{array}$ \\
\hline $\begin{array}{l}\text { 2-D cumulative mass } \\
\text { 3-D cumulative mass }\end{array}$ & F & FS & (see below) \\
$\begin{array}{l}\text { Sholl analysis } \\
\text { 2-D box counting } \\
\quad \text { coastline) }\end{array}$ & - & FS & \\
$\begin{array}{l}\text { 2-D box counting } \\
\text { (no coastline) }\end{array}$ & - & I S & \\
3-D box counting & - & F \\
\hline
\end{tabular}

' $F$ ' indicates significant differences at the $P<0.05$ level using the Fisher PLSD test, and ' $S$ ' indicates significant differences at the $P<0.05$ level using the Scheffé test. Tonic $W$ and phasic $W$ cells meet the Fisher PLSD test $(P<0.05)$, but the uncertainty in $P$ is large enough for these 2 cell populations that they may only be different at the $P=0.08$ level.

(no coastline). There was also a significant difference between the 2-D box-counting methods when examined with and without coastlines.

Although the absolute fractal dimension of a cell will vary according to the method used to compute it, the relative morphological complexity as measured by the fractal dimension will remain the same, irrespective of method. Thus, the $X$ cells show the highest fractal values, the tonic $W$ cells the intermediate values, and the phasic W cells the lowest values. We used the $95 \%$ confidence intervals as a test to see how we might classify cells by their fractal dimensions (Table 7). This classification procedure was $100 \%$ successful for $\mathrm{X}$ cells, but classified $20 \%$ of tonic $\mathrm{W}$ cells as phasic W cells and $40 \%$ of phasic W cells as tonic W cells.

Table 6

Comparison between different fractal methods and Sholl analysis using the Student's $t$ test (2-tailed)

\begin{tabular}{|c|c|c|c|}
\hline Method & $t$ valuc & $d t$ & $P$ \\
\hline 2-D C $\mathrm{m}_{\mathrm{m}}$ versus $3-\mathrm{DC}_{\mathrm{m}}$ & -4.05 & 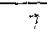 & $<0.005$ \\
\hline 2-D C $C_{m}$ versus Sholl & -1.16 & 14 & NS \\
\hline $2-D C_{m}$ versus $2-D B_{c}(C)$ & $24 ?$ & 10 & $<0.021$ \\
\hline 2-D C versus $2-D B_{c}$ (no $C$ ) & 6.59 & 19 & $<0,000$ \\
\hline 2-D C $\mathrm{C}_{\mathrm{m}}$ versus $3-\mathrm{D} \mathrm{B}_{\mathrm{c}}$ & -0.53 & $"$ & NS \\
\hline 3-D C $m$ versus Sholl & 0.04 & $\because$ & NS \\
\hline 3. $\mathrm{DC}_{\mathrm{m}}$ versus $2-\mathrm{D} \mathrm{B}_{\mathrm{c}}(\mathrm{C})$ & 2.51 & $?$ & NS \\
\hline $3-\mathrm{DC}_{\mathrm{m}}$ versus $2-\mathrm{D} \mathrm{B}_{\mathrm{c}}$ (no $\mathrm{C}$ ) & 4.51 & 3 & $<0.020$ \\
\hline 3-D C $C_{m}$ versus $3-D B_{c}$ & 0.26 & 7 & $\mathrm{NS}$ \\
\hline Sholl versus $2-D B_{c}(C)$ & 202 & 10 & NS \\
\hline Sholl versus 2-D B $B_{c}$ no $C$ ) & 3.36 & 14 & $<0,007$ \\
\hline Sholl versus $3 \cdot \mathrm{D} \mathrm{B}_{\mathrm{c}}$ & 0.38 & ? & NS \\
\hline 2-D B $B_{c}(C)$ versus $2-D B_{c}($ no $C)$ & 5.33 & $1 \%$ & $<0.000$ \\
\hline 2-D B $B_{c}(C)$ versus 3-D B & -0.78 & i & NS \\
\hline $2-D_{c}\left(\right.$ no $C$ ) versus $3-D B_{c}$ & -2.18 & 3 & NS \\
\hline
\end{tabular}

$C_{m}$, cumulative mass; $B_{c}$, box counting; $C$. coastline: no $C$, no coastline. 
Table 7

Percentage of cells classified as $\mathrm{X}$, tonic $\mathrm{W}$ or phasic $\mathrm{W}$ cells using the cumulative-mass method for fractal analysis

\begin{tabular}{llll}
\hline Classification & $\begin{array}{l}\mathrm{X} \text { cells } \\
\left(d_{\mathrm{f}}=1.72-1.77\right)\end{array}$ & $\begin{array}{l}\text { Tonic W cells } \\
\left(d_{\mathrm{f}}=1.58-1.64\right)\end{array}$ & $\begin{array}{l}\text { Phasic W cells } \\
\left(d_{\mathrm{f}}=1.32-1.70\right)\end{array}$ \\
\hline X cells & $100 \%$ & $0 \%$ & $0 \%$ \\
Tonic W cells & $0 \%$ & $80 \%$ & $20 \%$ \\
Phasic W cells & $0 \%$ & $40 \%$ & $60 \%$
\end{tabular}

Based on the indicated $95 \%$ confidence intervals for each cell type, $d_{\mathrm{f}}$ fractal dimension.

\subsection{Comparison of results of fractal and Sholl analyses}

In his anatomical studies of cortical neurons, Sholl (1953) determined the number of intersections of dendritic branches with a spherical shell per square micrometer versus the radius of the shell. He plotted the values either as $\log -\log (\log -\log$ Sholl analysis) or as log-linear (semi-log Sholl analysis). He used the slope of the graph $(k)$ to characterize the morphology of the neurons that he studied. Sholl used log-log analysis on apical dendrites and semi-log analysis on basal dendrites because only those analyses respectively produced linear results. Log-log Sholl analysis correlated best with 3-D box-counting fractal analysis (Spearman rank correlation coefficient (2-tailed) $r=0.884$, Table 8 ), but Sholl analysis showed large fluctuations in that the variation from cell to cell was much larger than that seen with fractal analysis. We were unable to relate semi-log Sholl analysis to fractal analysis because semi-log Sholl analysis of our neurons did not yield straight lines. There was therefore, no single slope to correlate with the slopes derived from fractal analysis.

We did a correlation analysis (Table 8 ) to determine the relationships or associations between all of our methods for analyzing neuronal complexity to learn if these methods were correlated with each other. There was a strong correlation between the 2-D cumulativemass method and both the 2-D box-counting methods $(P<0.01)$ and the 3-D cumulative-mass method $(P<$ $0.001)$. Although Sholl analysis showed a high noise level, it correlated well $(P<0.01)$ with the $3-\mathrm{D}$ boxcounting method. On the other hand, the 3-D cumula- tive-mass method only showed a significant correlation $(P<0.001)$ with the $2-\mathrm{D}$ cumulative-mass method.

\subsection{Curve fitting}

In order to calculate $d_{\mathrm{f}}$, it is necessary to fit a straight line to the graph of $\log M$ versus $\log r$. To minimize the errors introduced by this operation we used a fractal analysis method (cumulative mass) which did not add artifacts to the graph of $\log M$ versus log $r$. For instance, box counting can add a spurious nonlinearity to $\log M$ versus $\log r$ ( $\mathrm{Li}$ et al., 1989). 'Thus, it is prudent to measure $d_{\mathrm{f}}$ with more than one method. When we chose the part of the graph to analyze, we excluded those parts of the graph which were larger than the neuron, and those which were smaller than a single branch and so were indicating a 1-dimensional structure. We determined which parts of this graph to exclude by calculating the local slopes. Only the portion with constant local slopes was retained; and its slope was reported as the $d_{\mathrm{f}}$ of the neuron.

Calculating the local slope increases noise. A small amount of noise in the data is large compared to the distance between points, and this introduces a large variation in slope. In order to reduce the noise we calculated both the 2-point and 8-point local slopes (Fig. 5B,C). Two-point local slopes are the slopes of lines drawn through 2 successive data points, in contrast to 8-point local slopes which are the slope of a line fit to 8 successive data points using the leastsquares method. Note that although the 8-point local slopes greatly reduced the noise, we lost part of the linear section of the graph which we sought to analyze. The 8-point local slopes lose the linearity of the 2-point local slopes because sharp changes in local slope tend to be blunted due to averaging with their neighboring points and, thus, produce curves rather than straight line graphs with sharp inflection points. As one gets closer and closer to the region of low slope the averaging deflects the points further and further down (Fig. 5C). If there are regions of low slope on either side of the fractal region then the fractal region will be curved on both ends. Thus its linear appearance will be greatly

Table 8

Correlation analysis of different fractal methods and Sholl analysis using the Spearman rank correlation coefficient (2-tailed)

\begin{tabular}{|c|c|c|c|c|c|c|}
\hline & $2-\mathrm{DC}_{\mathrm{m}}$ & 3-D. $C_{m}$ & Sholl & 2-D B $(C)$ & 2-D B $B_{c}(C)$ & 3-D B \\
\hline $2-\mathrm{D} \mathrm{C}$ & 1.000 & $0.978^{* * *}(8)$ & $0.350(15)$ & $0.740^{* *}(11)$ & $0.796^{* *}(11)$ & $0.533(8)$ \\
\hline $3-\mathrm{D} \mathrm{C}_{\mathrm{m}}$ & $0.978^{* * *}(8)$ & 1.000 & $0.539(8)$ & $0.880(4)$ & $0.883(4)$ & $0.411(8)$ \\
\hline Sholl & $0.350(15)$ & $0.539(8)$ & 1.000 & $-0.047(11)$ & $0.280(11)$ & $0.884 * *(8)$ \\
\hline $2-\mathrm{D} \mathrm{B}(\mathrm{C})$ & $0.740^{* *}(11)$ & $0.880(4)$ & $-0.047(11)$ & 1.000 & $0.864 * * *(18)$ & $0.448(4)$ \\
\hline $2-B_{c}($ no $C)$ & $0.796^{* *}(11)$ & $0.883(4)$ & $0.280(11)$ & $0.864 * * *(18)$ & 1.000 & $0.482(4)$ \\
\hline 3-D B & $0.533(8)$ & $0.411(8)$ & $0.844^{* *}(8)$ & $0.448(4)$ & $0.482(4)$ & 1.000 \\
\hline
\end{tabular}

$\mathrm{C}_{\mathrm{m}}$, cumulative mass; $\mathrm{B}_{\mathrm{c}}$, box counting; $\mathrm{C}$, coastline; no $\mathrm{C}$, no coastline.

${ }^{*} P<0.05,{ }^{* *} P<0.01$ and ${ }^{* * *} P<0.001$; number of cells in parentheses. 
5

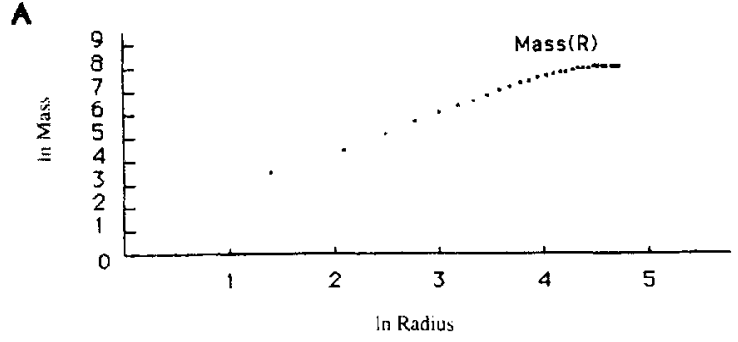

B

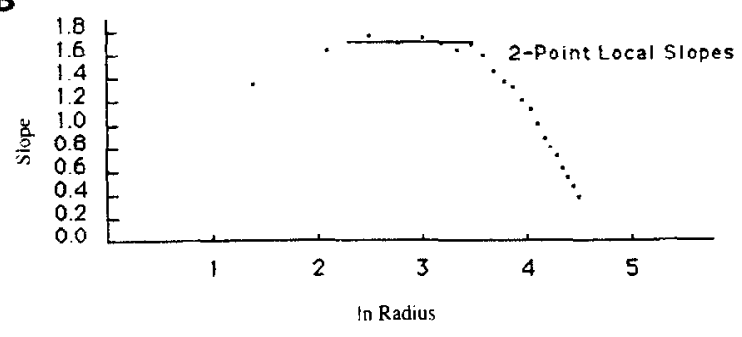

c

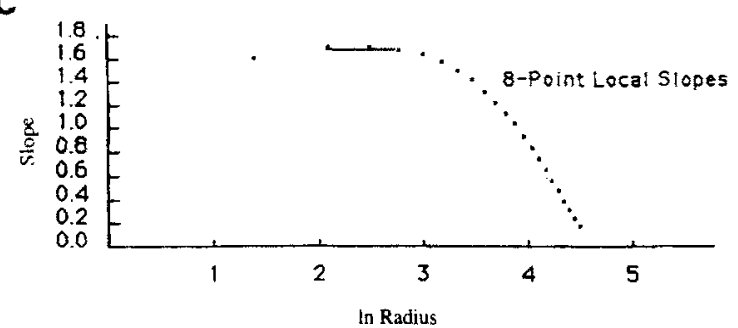

Fig. 5. Log-log plot of mass versus radius (A), and the associated semi-log plots of 2-point (B) and 8-point (C) local slopes of plot A. A: the graph of $\log M$ versus $\log r$ from which the local slope graphs were derived. The solid lines in $B$ and $C$ indicate the linear section of each local slope plot. Note that the 8-point local slope plot $(C)$ is both smoother and has a shorter linear section than the 2-point local slope plot (B).

diminished. Though 8-points slopes might be useful if there were many points on the graph of $\log M$ versus $\log r$, Fig. 5a demonstrates a situation where there are not enough points to use the 8-point slope without introducing errors into the slopes.

One can calculate several least-squares lines fitting the $\log M$ versus $\log r$ data since there are some small variations in slope in different portions of the linear region. Even if the confidence level is $99 \%$, the variation among these slopes can be more than $5 \%$ since they are calculated from different parts of the linear region and, therefore, different data sets.

\section{Discussion}

\subsection{Underlying basis of fractal dimension}

In this report we compare several different methods for calculating the fractal dimensions of neurons. Using these methods, we analyzed different physiologically characterized cell types and found some significantly different $d_{\mathrm{f}}$ s (see Table 3 ). In a previous study (Caserta et al., 1990), we found that the fractal dimensions $\left(d_{s} s\right)$ of neurons (1.68 SD 0.15) were close to that of DLA (1.70 SD 0.10). However, we recognized and others have shown (Montague and Friedlander, 1991; Smith et ai., 1991) that the $d_{f} \mathrm{~s}$ of some neurons fall below the DLA range (i.e., phasic W cells; $d_{1}=1.52$ SD 0.15 ) and some are above the DLA range (i.e., $\mathrm{X}$ cells; $d_{f}=1.75$ SD 0.02). The importance of DLA is that it suggests an underlying growth process which might be one possible starting point for modeling neurite outgrowth and neuronal pattern formation for some cell types. If neuronal growth processes really have a DI A component, then the variation in measured $d_{f}$ might be explained by incorporating additional features into DLA, such as the retraction of branches (Meakin, 1991) or a non-linear response to growth-stimulating chemical or electrical (Caserta et al., 1992) gradients. Both modifications will produce a wide range of $d_{\mathrm{f}}$ s. Such branch retraction and chemotropism are known to be involved in neuronal growth mechanisms (Lockerbie, 1987). However, even if neurons had exactly the same $d_{f}$ as DLA, it would not prove that neurons grow by a DLA process. Two fractals can have the same $d_{1}$ and grow by different mechanisms. Even though percolation clusters and DLA grow by different rules, both have a $d_{f}$ of 2.5 when grown in a 3-D space (Meakin. 1988).

\subsection{Methodology of fractal analysis}

In order to make fractal analysis more meaningful, we must first define 'fractal'. A natural fractal is defined as an object in nature which grows more massive, as we measure the mass $(M)$ in larger and larger ficlds of view $(r)$, in such a way that the graph of $\log M$ versus $\log r$ is linear over one or more decades. The above definition does not include any requirement for a fractal growth rule. For instance, DLA, a very wellstudied natural fractal, has no fractal growth rule, but on the contrary grows according to a non-scale-dependent, non-recursive rule (i.e., growth velocity is proportional to the chemical concentration on the boundary of the DLA cluster). Thus, though neurons seem to be fractal, no fractal growth rule is required in any theory of their morphological development. In contrast, a mathematical fractal is a theoretical abstraction containing an infinite range of feature sizes and displaying $\log M$ versus $\log r$ linearity over an infinite number of decades. While both natural and mathematical fractals are linear in $\log M$ versus $\log r$, the short interval of linearity in natural fractals presents additional difficulties in determining linearity and fractality. By contrast, mathematical fractals can be realized on a computer with an extremely long linear interval. 
For any natural fractal which is fractal over at least 1 decade, there are many methods available for counting the mass of the object or measuring its coastline. Three methods of measuring neuronal coastline length were compared by Smith et al. (1989). The methods were caliper, disk, and grid. Caliper measures the coastline with straight lines. Disk measures the coastline with disks. Grid measures the coastline by placing grids of different grid sizes on the coastline and counting the number of non-empty boxes (which is the same as box counting the coastline). Measuring the coastlines of neurons and other objects with calipers of various sized openings was found to produce less linear graphs by Smith et al. (1989) than the other methods. By analyzing only the coastline or perimeter of a neuron, instead of the space inside the coastline we obtained a higher fractal dimension. This was due to the fact that the coastline of a neurite is thicker than the original neurite and looks 2-D on small length scales, as opposed to the original neurite which is one dimensional on small length scales. Thus, the influence of the coastline is to increase the value obtained for $d_{\mathrm{f}}$ which is calculated over a range of length scales.

Mass methods, such as the cumulative-mass method, have the advantage over coastline methods in that they measure the $d_{f}$ of the neuron directly, rather than measuring the $d_{\mathrm{f}}$ of its coastline and then trying to relate it to the $d_{\mathrm{f}}$ of the neuron. Morigiwa et al. (1989) and Montague and Friedlander (1989) used mass-box counting to study the $\log M$ versus $\log r$ relationship of neurons. In the present report, we found the $d_{\mathrm{f}} \mathrm{s}$ of neurons calculated by box counting to be lower than that by the cumulative-mass method. This is true also for DLA where box counting produced a fractal dimension of $1.57 \mathrm{SD} 0.05$ ( $\mathrm{Li}$ et al., 1989), as opposed to the well established value of $1.71 \mathrm{SD} 0.02$ (Meakin, 1983). The cumulative-mass method has the disadvantage of requiring a computer with the floating-point instruction speed of a mainframe as opposed to box counting, which runs acceptably fast on a PC. However, the error due to the small finite size of objects is much less for the cumulative-mass method than for box counting. Similarly, the significance of differences in $d_{\mathrm{f}} \mathrm{s}$ for different physiological types of neurons is higher using the cumulative-mass method than the significance using box counting in our study.

When using the cumulative-mass method, it is necessary to choose a radius within which the centers of the cumulative masses will be placed. For an object like a DLA cluster, these centers are usually taken within the radius of gyration of the DLA cluster. The radius of gyration of an object is the square root of the sum of the squares of the radii from the center of mass to all the points on the object, divided by the square root of the number of points. This choice of centers specifically excludes the periphery of the object in order not to be influenced by the low $d_{\mathrm{f}}$ of the 'unfinished' outer regions (A. Coniglio, personal communication). However, it is possible to take the cumulative mass centers at all points on the structure, sampling both the center and the periphery (Meakin, 1992). The choice of whether to include the periphery must be based on whether the periphery is judged to be a 'finished' part of the whole fractal. 'Unfinished' connotes the idea that the outer regions of a fractal may have a different fractal dimension than the inner region. Therefore, in the present case, we chose centers within the radius of gyration because the branching patterns of the neurons we analyzed were not 'finished'. Although the neurons were adult and probably not actively growing, their outer branches, in terms of fractal analysis, were not 'finished'. To be 'finished', in terms of fractal analysis, the outer branches would have to have the same fractal dimension as the inner branches. The inclusion of the outer branches incorrectly lowers the $d_{\mathrm{f}}$ of the neuron and produces errors due to the large variability of the contribution of the outer branches. Natural fractals like neurons are often not 'finished', as opposed to abstract, mathematical fractals which are forced to be 'finished', by definition (i.e., all parts of the fractal are constructed with the same fractal dimension).

When performing fractal analysis, it is good to note that different methods work best for different fractals. Cumulative mass works well for DLA and objects that grow radially from their center but not well for fractals like the Sierpinski carpet which has no obvious growth center. Box counting works well for the Sierpinski carpet but not for DLA. Thus, even though a method works well with a fractal of known $d_{\mathrm{f}}$, it may not work for all fractals. It is very important to take into account the contribution of the periphery of the object under analysis. The periphery may have a different $d_{\mathrm{f}}$ than the rest of the object (A. Coniglio, personal communication). This part of the object must be ignored to calculate the correct $d_{\mathrm{f}}$. This is particularly important if the object has few length scales in it because the periphery's contribution would dominate. We used a cumulative-mass method which ignores the periphery of the neuron and so removes any errors associated with it. Our box-counting methods did not remove the periphery. Thus, we believe that the different $d_{\mathrm{f}} \mathrm{s}$ obtained using the cumulative-mass and box-counting methods is partially due to the different treatments of the periphery.

\subsection{Analysis of characterized cells}

With our statistical analysis of groups of cells of different physiological typcs, the significance of differences in $d_{\mathrm{f}}$ for different physiological types of cells can be determined. The methods of analysis used here 
demonstrated that the 3 types of cat retinal ganglion cells we analyzed differed in their fractal dimensions. This lends support to the notion that these cell types do, in fact, represent populations of neurons that can be discriminated from one another. Stanford (1987a) presented data that strongly suggested that cat retinal W cells can be subdivided into at least 2 subtypes based on both morphological and physiological criteria. The differences in the fractal dimensions found here for phasic and tonic W cells supports this suggestion. In the present analysis, we found that the fractal dimensions of tonic $W$ cells had values more similar to that of $X$ cells. These data, and the similarities in the soma sizes of tonic $\mathrm{W}$ cells and $\mathrm{X}$ cells previously reported (Stanford, 1987a), indicate that, at least morphologically, tonic $\mathrm{W}$ cells are more similar to $\mathrm{X}$ cells than to the phasic W cells. The phasic W cells analyzed here had the most variability in fractal dimensions (Fig. 4), indicating that these cat retinal ganglion cells are the most heterogeneous. It is possible that some of these morphologically diverse phasic $\mathrm{W}$ cells may represent the ganglion cells in the cat retina that have been reported to have unusual response features such as suppressed by contrast cells, local edge detectors, etc. (Cleland and Levick, 1974; Stone and Fukuda, 1974). Differences in the fractal dimensions of retinal ganglion cells have been previously reported by Morigiwa et al. (1989), who described significant differences in the fractal dimensions of ON- and OFF-type ganglion cells in the cat retina.

The 2-D cumulative-mass method was the best method for differentiating between $X$ cells and tonic $W$ cells, and $\mathrm{X}$ cells and phasic $\mathrm{W}$ cells in comparison with either Sholl analysis or 2-D box counting of the neurons with or without inclusion of the coastline (Table 3). More study is required to precisely determine the reason for the difference in performance of these 4 methods.

\subsection{Comparison of fractal and Sholl analysis results}

The 2 different methods of Sholl analysis, log-log and semi-log, have been applied previously without a clear basis as to which to chnose (Lima and Coimbra, 1989). Of the 2 methods, only log-log Sholl analysis correlates with fractal analysis for the cells that we analyzed. We correlated the outputs of all our methods (Table 8 ) and found that the 3-D box counting fractal analysis correlated best with log-log Sholl analysis (Spearman rank correlation coefficient (2-tailed) $r=$ 0.884 ). One aspect where the methods differed was that Sholl analysis used only one center from which to calculate distances to pixels, while fractal analysis used all points on the neuron within the radius of gyration as centers. By examining all points, fractal analysis (cumulative-mass method) can average out noise, which produces a more accurate representation of the neuron. However, Sholl analysis is centered on only one point on the neuron's structure, and therefore, random noise is introduced based on which part of the random structure is located at the center. Sholl analysis had the highest noise level and because of this high noise level it is perhaps the less useful method.

\subsection{3-Dimensional analysis}

Most previous work has been limited to 2-D fractal analysis of cells whose physiological type was not directly determined (Montague and Friedlander, 1989, 1991) or cells which were characterized physiologically on the basis of their anatomy (Morigiwa et al., 1989). Ours is the first complete 2-D and 3-D analysis of physiologically characterized neurons. We found 3-D analysis to be possible although the retinal ganglion cells we analyzed are somewhat unique in that they are relatively 2-D. This is due to the fact that all retinal ganglion cells in the cat receive their synaptic input in the inner plexiform layer and are constrained to have their dendrites ramify in that layer of the retina. However, the fact that the $d_{\mathrm{f}} \mathrm{s}$ in 3 dimensions were higher is consistent with the higher theoretical values of known fractals in 3 versus 2 dimensions (DLA, 3-D $d_{\mathrm{f}}=2.5$. 2-D $d_{\mathrm{f}}=1.71$ ) (Meakin, 1988). It is likely that neurons which arborize more completely in 3 dimensions will have $d_{\mathrm{f}} \mathrm{s}$ closer to 2.5 . Since 3-D analysis showed significant differences in $d_{i}$ for these relatively 2-D cells, 3-D analysis might be even more revealing if applied to more truly 3-D cells.

In conclusion, there are many methods for fractal analysis and no one method is superior for analysis of all fractal objects. When choosing a method, one must consider that the best method may be one (e.g. cumulative mass) that works well on known natural fractals like DLA that have similar properties to neurons, such as radial growth from the center and an 'unfinished' periphery. Methods (e.g., box counting) which work well on mathematical fractals like the Sierpinski carpet may not work as well for natural fractals. Sholl analysis is similar to fractal analysis, but usually is subject to relatively more random noise. The three types of cat retinal ganglion cells that we analyzed using the 2-D cumulative-mass method differed in their fractal dimensions, lending support to the idea that these types of cells represent populations of cells which can be discriminated from one another on the basis of their morphological, as well as physiological characteristics. By calculating statistics comparing groups of cells of different physiological types, we were able to determine the significance of these differences in fractal dimension. We extended sand hox analysis to 3 dimensions and demonstrated its ability to find significant differences between the average fractal dimensions of 
these cell types. Therefore, we believe fractal dimension can be a useful parameter for the morphometric characterization of the apparent complexity of the dendritic arborizations of neurons in 2 and 3 dimensions.

\section{Acknowledgements}

The work at Boston University was supported in part by grants from the NSF, ONR, and NEI (W.D.E., EY04785). The work at the University of Wisconsin was supported by a grant from NEI (L.R.S., EY04977). We thank Professor P. Meakin for many helpful suggestions on fractal analysis, Professor B. Chasan for constructive criticism and Professor M. Shann for help with statistical analysis. The computer programs for fractal analysis and Sholl analysis are available from $\mathrm{F}$. Caserta, Physics Dept., Boston University, Boston, MA 02215 (Bitnet address: caserta@buphy.bu.edu).

\section{References}

Adams, J.C. (1981) Heavy metal intensification of DAB-based HRP reaction product, J. Histochem. Cytochem., 29: 775.

Caserta, F., Stanley, H.E., Eldred, W.D., Daccord, G., Hausman, R.E., and Nittman, J. (1990) Physical mechanisms underlying neurite outgrowth: A quantitative analysis of neuronal shape, Phys. Rev. Lett., 64: 95-98.

Caserta, F., Hausman, R.E., Eldred, W.D., Kimmel, C., and Stanley, H.E. (1992) Effect of viscosity on neurite outgrowth and fractal dimension. Neurosci. Lett., 136: 198-202.

Daccord, G. (1987) Chemical dissolution of a porous medium by a reactive fluid, Phys. Rev. Lett., 58: 479-482.

Daccord, G. and Lenormand, R. (1987) Fractal patterns from chemical dissolution, Nature, 325: 41-43.

Daccord, G. Nittmann, J. and Stanley, H.E. (1986) Radial viscous fingers and diffusion limited aggregation: fractal dimension and growth sites, Phys. Rev. Lett., 56: 336-339.

Kleinfeld, D., Raccuia-Bchling, F., and Blonder, G.E. (1990) Comment on 'Physical mechanisms underlying neurite outgrowth: A quantitative analysis of neuronal shape', Phys. Rev. Lett., 65: 3064.
Li, G., Sander, L.M., Meakin, P. (1989) Comment on 'Self-similarity of diffusion-limited aggregates and electrodeposition clusters', Phys. Rev. Lett., 63: 1322.

Lima, D. and Coimbra, A. (1989) Morphological types of spinomesencephalic neurons in the marginal zone (lamina I) of the rat spinal cord, as shown after retrograde labelling with cholera toxin subunit B, J. Comp. Neurol., 279: 327-339.

Lockerbie, R.O. (1987) The neuronal growth cone: a review of its locomotory, navigational, and target recognition capabilities, Neuroscience, 20: 719-729.

Meakin, P. (1983) Diffusion-controlled cluster formation in 2-6-dimensional space, Phys. Rev., A27: 1495.

Meakin, P. (1988) The growth of fractal aggregates and their fractal measures. In: C. Domb and J.L. Lebowitz (Eds.), Phase Transitions and Critical Phenomena, Vol. 12, Academic, New York, pp. 336-489.

Meakin, P., and Feder, J. (1991) Growth of adaptive networks in a DLA model, Phys. Rev., A44: 5104.

Morigiwa, K., Tauchi, M., and Fukuda, Y. (1989) Fractal analysis of ganglion cell dendritic branching patterns of the rat and cat retinae, Neurosci. Res., Suppl. 10: \$131-S140.

Montague, P.R., and Friedlander, M.J. (1989) Expression of an intrinsic growth strategy by mammalian retinal neurons, Proc. Natl. Acad. Sci., 86: 7223-7227.

Montague, P.R., and Friedlander, M.J. (1991) Morphogenesis and territorial coverage by isolated mammalian retinal ganglion cells, J. Neurosci., 11: 1440-1454.

Nittmann, J., Daccord, G. and Stanley, H.E. (1985) Fractal growth of viscous fingers: a quantitative characterization of a fluid instability phenomenon, Nature, 314: 141-144.

Sholl, D.A. (1953) Dendritic organization in the neurons of the visual and motor cortices of the cat, J. Anat. (Lond.), 87: 387-406.

Smith, T.G., Marks, W.B., Lange, G.D., Sheriff, W.H., and Neale, E.A. (1989) A fractal analysis of cell images, J. Neurosci. Methods, 27: 173-180.

Smith, T.G., Behar, T.N., Lange, G.D., Marks, W.B. and Sheriff, W.H. Jr. (1991) A fractal analysis of cultured rat optic nerve glial growth and differentiation, Neuroscience, 41: 159-166.

Stanford, L.R. (1987a) W Cells in the cat retina: correlated morphological and physiological evidence for two distinct classes, J. Neurophys., 57: 218-244.

Stanford, L.R. (1987b) X-cells in the cat retina: relationships between the morphology and physiology of a class of cat retinal ganglion cells, J. Neurophys., 58: 940-964.

Tel, T., Fulop, A. and Vicsek, T. (1989) Determination of fractal dimensions for geometric multifractals, Physica A, 159: 155-166.

Vicsek, T. (1989) Fractal Growth Phenomena, World Scientific, Singapore, pp. 80-81. 